\title{
"ANNUNCIARE AI POVERI IL LIETO MESSAGGIO": LA SCELTA PREFERENZIALE DEI POVERI OGGI
}

\author{
Mons. Giancarlo Perego*
}

\section{Premessa}

Papa Francesco ha ricordato, a parole e gesti, ancora una volta, come la Chiesa è chiamata ad essere povera e dei poveri. Non si tratta semplicemente della riabilitazione di categorie sociologiche, ma di un annuncio evangelico che riprende il messaggio cristologico e della storia della salvezza. Come sottolinea il recente documento Accogliere Cristo nei rifugiati e nelle persone forzatamente sradicate in relazione ai migranti, "identificandosi con lo straniero, Gesù Cristo ha messo in luce quale dovrebbe essere il modo cristiano di considerare e trattare lo straniero. 'Negli «stranieri» la Chiesa vede Cristo che «mette la sua tenda in mezzo a noi» (cfr Gv 1,14) e che «bussa alla nostra porta» (cfr Ap 3,20)' (EMCC 101)" (n. 22). Collocato, poi, questo messaggio in questo tempo di crisi, corrisponde anche a uno 'stile di vita' che dice non solo a parole, ma con i fatti la ricerca di un bene comune che passa necessariamente attraverso la condivisione e la redistribuzione dei beni, la sobrietà.

\section{Dal Cristo povero alla Chiesa dei poveri: dalla redenzione all'evangelizzazione}

\section{Le parole e i gesti di Gesù: la cristologia delle relazioni}

La storia di Gesù è una storia di parole, ma anche di relazioni. L'evangelista Matteo costruisce il suo Vangelo su cinque grandi discorsi (sull'amore, il discorso escatologico, il discorso in parabole, il discorso apostolico, il discorso sulla Chiesa). Cinque discorsi che dicono la necessità dell'ascolto di Gesù, anzitutto; ma cinque discorsi che dicono anche l'importanza dell'ascolto: come strada dell'amore, come luogo di giudizio, come stile apostolico, come vissuto della Chiesa. Marco e Giovanni sottolineano molto il valore di questi

* Direttore Generale della Fondazione Migrantes, insegnante di Teologia Dogmatica presso la Facoltà di Scienze delle Comunicazione LUMSA. E-mail: perego@migrantes.it. Roma/Italia. 
incontri e relazioni, soprattutto con gli ultimi: i bambini e le donne, gli stranieri, i malati, i samaritani. Non ascoltare la Parola e le parole degli uomini, non creare relazioni significa non maturare nella fede; non incontrare le persone, soprattutto i più poveri, significa non camminare sulle strade della carità, non vivere di speranza, perchè si scade nell'abitudinarietà. L'ascolto e la relazione in Gesù e, di conseguenza, nella Chiesa è la prima strada dell"affetto', che non è un semplice sentimento, ma un cammino di maturazione della consapevolezza dell' 'ad-factus', cioè dell'essere 'fatto per'.

\section{L'ambivalenza o equivocità del concetto di povertà}

Il concetto di 'povero' nella Bibbia non ha un senso univoco, ma equivoco. Povero nella Bibbia significa: cattivo, malvagio (peccato); afflitto, perseguitato; umile, disponibile di fronte al Signore (grazia); I'orfano, la vedova, lo straniero (Dt 24,17-18). Nella cultura moderna povero significa: chi non ha un reddito minimo, manca di qualcosa di vitale; chi chiede aiuto; chi è solo; chi è escluso; chi non è riconosciuto. Saper individuare il volto di 'chi' è povero, anche con metodo e con strumenti scientifici di rilevazione, con una particolare attenzione al territorio, non dimenticando, però, il valore essenziale della relazione e della condivisione, è un compito importante nel lavoro pastorale.

\section{La scelta dei poveri della prima comunità cristiana}

La prima comunità cristiana riconosce di essere 'libera davvero' dalla rinuncia-condivisione dei beni (libertà da mammona) e dalla libertà dal potere. $E^{\prime}$ vero che il vocabolario sulla povertà negli Atti è ridotto rispetto ai Vangeli e a Paolo; così pure i termini della solidarietà sono concentrati nei sommari. Tuttavia, sia in Paolo che negli Atti, il termine 'koinonia' esprime la storia nuova della comunità, coniugata in alcune scelte: la comunione dei beni (At 2,42-47; 4,32-35), il servizio delle mense (At 6,1-6), la colletta antiochena per la chiesa della Giudea (At 11, 27-30; 12,25); la grande colletta per Gerusalemme (Gal 2,10; 1Cor 16,1-4; 2Cor 8-9); Rm 15,25-32).

\section{Evangelizzazione e diaconia}

\section{L'evangelizzazione è testimonianza}

L'8 dicembre 1975 Paolo VI promulgava l'esortazione apostolica 'Evangelii Nuntiandi' (EN), uno dei documenti più importanti e discussi del suo Pontificato, come recentemente un Colloquio internazionale di studi organizzato dall'Istituto Paolo VI di Brescia ha rilevato

\footnotetext{
${ }^{1}$ L'esortazione apostolica di Paolo VI 'Evangelii nuntiandi'. Soria, contenuti, ricezione. Brescia-Roma: Studium, 1998.
} 
"Annunciare ai poveri il lieto messaggio": la scelta preferenziale dei poveri oggi

In quel documento, nato tra opposte tendenze di chi riduceva l'evangelizzazione alla promozione umana - cadendo in una 'nuova secolarizzazione' - e di chi escludeva la promozione umana dall'evangelizzazione, si affermava che tra evangelizzazione e promozione umana esistono legami profondi.

Al n. 24 del documento, Paolo VI scriverà che "I'evangelizzazione è un processo complesso e dagli elementi vari: rinnovamento dell'umanità, testimonianza, annuncio esplicito, adesione del cuore, ingresso nella comunità, accoglienza dei segni, iniziative diapostolato". "L'evangelizzazione-aggiungeva Paolo VI - non si esaurisce nella predicazione e nell'insegnamento di una dottrina. Essa deve raggiungere la vita" (EN 47). Luogo dell'evangelizzazione è la Chiesa: compito fondamentale della Chiesa è l'evangelizzazione. La Chiesa evangelizza nella misura in cui "ascolta di continuo ciò che deve credere, le ragioni della sua speranza, il comandamento nuovo dell'amore" (EN 15).

Fede, speranza e carità non sono solo le 'virtù' del singolo credente, ma anche le 'virtù' di una Chiesa che evangelizza oggi. In forza di questa visione complementare, dinamica dell'evangelizzazione della Chiesa e nella Chiesa, Paolo VI arriva ad affermare che la prima forma di annuncio è la testimonianza. In un mondo ricco di messaggi, in parole e immagini, che talora disorientano, scandalizzano, l'uomo cerca "più volentieri i testimoni che i maestri... o se ascolta i maestri lo fa perché sono dei testimoni" (EN 41).

La Chiesa che evangelizza è una Chiesa di "testimoni", di "testimonianze": di "profeti" e di "segni" che incarnano in maniera nuova una cultura e dei tempi. La Chiesa della testimonianza è una Chiesa che "ascolta e custodisce la Parola e la confronta con le parole degli uomini, che custodisce e ascolta". E' il duplice primato: il Primato della Parola e la scelta preferenziale dei poveri. $\mathrm{E}^{\prime}$ anche il senso della circolarità tra liturgia, catechesi e carità. La Chiesa che testimonia è una Chiesa che osserva e valuta, ragiona sulle tragedie e sulle possibilità umane per costruire un futuro, per sperare. La Chiesa che testimonia è una Chiesa che riascolta, nelle parole e nei gesti di Gesù, una storia d'amore e la traduce in una storia di comunione fraterna, sempre in maniera originale.

\section{Le qualità dell'annuncio del Vangelo ai poveri}

E' importante sottolineare le diverse e necessarie 'qualità' dell'annuncio cristiano.

- Qualità cristologica dell'annuncio ai poveri: il legame tra redenzione e evangelizzazione, tra parole-gesto-croce e le parole-gesti-sofferenze che raccontano e realizzano nella storia e nella vita della Chiesa la salvezza. 
- Qualità simbolica dell'annuncio ai poveri: è nell'incontro tra Dio e I'uomo, a partire dal sacramento dell'Eucarestia, che siamo in grado di narrare ed realizzare l'oggi della salvezza. "Le ombre" della storia dell'uomo non impediscono questa qualità simbolica.

- Qualità pellegrinante o missionaria dell'annuncio ai poveri: non è un annuncio cattedratico, ma costruito nel confronto, nei laboratori, nella costruzione di itinerari di vita per i ragazzi e i giovani, per i fidanzati e gli sposi, per gli ammalati e per chi sta morendo, per i migranti e i rifugiati, nel percorrere un tratto di strada insieme, nella condivisione.

- Qualità relazionale-dialogica dell'annuncio ai poveri: è un annuncio non semplicemente costruito su paradossi e contrapposizioni, ma nella relazione, nel dialogo, nella proposta, nel rispetto dei tempi e dei dubbi di chi cresce, ha sbagliato, è smarrito.

- Qualità povera, dei mezzi poveri e privi di ogni prepotenza dell'annuncio ai poveri. E' la qualità del dono, più che della pretesa, dell'offrire più che del consumare.

- Qualità sofferente dell'annuncio ai poveri. Il povero soffre, è smarrito, è solo: aprire uno spazio nel cuore è soffrire, ma soffrire per qualcosa e per qualcuno e non per niente. Il valore della sofferenza nasce dalla sua destinazione.

- Qualità riformatrice nell'annuncio ai poveri: I'annuncio ai poveri, cambia la Chiesa, la spoglia non delle strutture - cadremmo nel rischio reiterato nella storia di spiritualismo! - ma delle infrastrutture o di paure o di falsi moralismi che impoveriscono le parole e le relazioni della Chiesa, facendole perdere la sua "virilità", direbbe Rahner.

La rilevanza di queste otto 'qualità' dell'annuncio cristiano mostrano chiaramente la connessione, la stretta relazione, I'osmosi che esiste tra celebrazione, annuncio e testimonianza: non esiste un 'vangelo della carità' se non dentro la relazione stretta tra queste tre dimensioni. Non s'impara la carità senza l'incontro con il Cristo povero nella parola, nell'eucarestia, nei poveri.

\section{La Chiesa dei poveri}

A partire da una rinnovata cristologia, attorno e durante il Concilio Vaticano II, alcuni teologi e pastori - quali il Paul Gauthier e Yves Congar, il cardinale Lercaro, don Mazzolari e don Milani -, avevano sollecitato una riflessione sulla povertà nella e della Chiesa e sull'annuncio ai poveri², tale da

${ }^{2}$ DUPONT, jacques. La Chiesa e la povertà. In La Chiesa del Vaticano II. Firenze: Vallardi, 1965, p. 387-418. 
arrivare a proporre come tema per il Concilio "I'evangelizzazione dei poveri" ${ }^{3}$. A questa proposta teologi e pastori erano arrivati a partire da due considerazioni pastorali: II mondo operaio ha bisogno di essere rievangelizzato; due terzi del mondo, fatto di poveri, reclama un uso diverso dei beni, in senso fraterno. Il Concilio non adotterà l'immagine della 'Chiesa dei poveri', forse perché ambigua, ma aiuterà a richiamare due aspetti qualitativi importanti attorno al tema della 'povertà': la condizione di privilegio dei poveri nel raccogliere I'annuncio evangelico e il collegamento tra povertà e storia di Gesù, così come emerge da Lc 4,18-21, il testo di Luca che riprende e rilegge Is 61,1-3. Scriverà il Concilio, nella Lumen Gentium, al n. 8:

Come Cristo ha compiuto la sua opera di redenzione attraverso la povertà e le persecuzioni, così pure la Chiesa è chiamata a prendere la stessa via per comunicare agli uomini i frutti della salvezza". "Gesù Cristo 'sussistendo nella natura di Dio...spogliò se stesso, prendendo la natura di un servo" (Fil. 2,6-7) e per noi "da ricco che egli era si fece povero" (2Cor 8,9): così anche la Chiesa, quantunque per compiere la sua missione abbia mezzi umani, non è costituita per cercare la gloria della terra, bensì per far conoscere, anche con il suo esempio, I'umiltà e l'abnegazione". "Cristo è stato inviato dal Padre "a dare la buona novella ai poveri, a guarire quelli che hanno il cuore contrito" (LC. 4,18), a "cercare e salvare ciò che era perduto" (Lc 10,10): così pure la Chiesa circonda di affettuosa cura quanti sono afflitti dall'umana debolezza, anzi riconosce nei poveri e nei sofferenti l'immagine del suo fondatore povero e sofferente, si premura di sollevarne l'indigenza, e in loro intende di servire a Cristo". "Ma mentre Cristo, 'santo, innocente, immacolato' (Eb 7,26), non conobbe il peccato $(2$ Cor 5,21$)$, ma venne allo scopo di espiare i soli peccati del popolo (Eb 2,17), la chiesa che comprende nel suo seno i peccatori, santa insieme e sempre bisognosa di purificazione, mai tralascia la penitenza e il suo rinnovamento". "La Chiesa 'prosegue il suo pellegrinaggio fra le persecuzioni del mondo e le consolazioni di Dio', annunziando la passione e la morte del Signore fino a che Egli venga (1Cor 11,26). Dalla forza del Signore risuscitato trova forza per vincere con pazienza e amore le sue interne ed esterne afflizioni e difficoltà, e per svelare al mondo, con fedeltà, anche se sotto ombre, il mistero del Signore, fino a che alla fine dei tempi sarà manifestato nella pienezza della sua luce.

${ }^{3}$ COLOMBO, Giuseppe. "Evangelizzare pauperibus". Riflessione teologica. In ASSOCIAZIONE BIBLICA ITALIANA. Evangelizzare pauperibus. Atti della XXIV settimana biblica. Brescia: Paideia, 1978, p. 29-36. 


\section{La scelta preferenziale per i poveri nella comunità cristiana di oggi}

\section{a. Chiarificazione dei termini}

$\mathrm{E}^{\prime}$ anzitutto indispensabile partire dalla chiarificazione dei termini: scelta, preferenziale, poveri. Per "scelta" intendiamo un gesto maturo, personale, consapevole, definitivo; un gesto libero, conseguenza di una vocazione, cioè di una chiamata da parte di Gesù Cristo (sequela Christi), secondo lo stile del Figlio di Dio, il quale passò in mezzo a noi beneficando tutti coloro che confidavano in Lui, soprattutto i più poveri e gli ammalati. Un gesto carico di responsabilità, non improvviso, non parziale e neppure generato semplicemente dalle situazioni, anche se ad esse legato. Con "preferenziale" indichiamo una scelta non esclusiva, nè escludente qualcuno. Diversamente, sarebbe una scelta non cattolica, perchè verrebbe meno il dato universalistico (andare in tutto il mondo, da tutti gli uomini). I "poveri" sono individuati a partire da tre connotazioni: da un punto di vista morale sono gli umili, i semplici; da un punto di vista sociale sono i piccoli, gli indifesi, i soli, i perseguitati; da un punto di vista materiale sono coloro senza mezzi necessari per vivere, i miseri.

\section{b. L'annuncio e la scelta per i poveri nel Magistero e nella pastorale della Chiesa}

Ci domandiamo come tale 'scelta' è stata assunta nella pastorale della Chiesa - della Chiesa italiana in particolare.

Il Concilio Vaticano II, con la Lumen Gentium al n. 8 offre un testo fondamentale. Dal testo conciliare si evince che la scelta per i poveri è una chiamata che non trova tanto una giustificazione nella realtà storico-sociale, quanto nel Mistero dell'Incarnazione, Passione e Morte, Risurrezione di Gesù Cristo e, di conseguenza, nel Mistero della Chiesa. Inoltre, dal testo si deduce che tale scelta porta con sè uno stile di comunicazione del Vangelo, uno stile di evangelizzazione. Nella stessa linea si muove l'Evangelii Nuntiandi di Paolo VI, la quale sottolinea la necessità di parlare della liberazione e promozione dell'uomo, soprattutto povero e indifeso, a partire dalla visione dell'uomo che Cristo ha manifestato (cf. n.31-38); al tempo stesso sottolinea il valore della testimonianza di povertà nella Chiesa. Il documento della Congregazione della dottrina della fede Libertatis coscientiae, del 1986, al n. 68 riprende il tema della scelta preferenziale dei poveri come non esclusiva. Nella Sollicitudo rei socialis di Giovanni Paolo II, nel 1988, parla dell' "amore preferenziale per i poveri" (n. 42).

La lettera apostolica di Giovanni Paolo II, al termine del Giubileo del 2000, 'Novo Millennio ineunte', riprendeva con molta precisione tale scelta 
"Annunciare ai poveri il lieto messaggio": la scelta preferenziale dei poveri oggi

in un paragrafo del n. 49: "Certo, non va dimenticato che nessuno può essere escluso dal nostro amore, dal momento che 'con l'incarnazione il Figlio di Dio si è unito in certo modo a ogni uomo'.

\section{L'evangelizzazione come testimonianza in parrocchia}

La nuova evangelizzazione chiede un' immagine di Chiesa "nuova, esemplare" - come si è ricordato al Convegno di Palermo e nella nota pastorale della CEI "Con il dono della carità dentro la storia", al n. 2:

una Chiesa che ascolta e medita la Parola, perché non c'è rinnovamento, anche sociale, che non parta dalla contemplazione; una Chiesa che celebra la liturgia con canti festosi e gesti semplici, ma significativi;

una Chiesa unita nell'attiva partecipazione di pastori, teologi, religiosi, laici, uomini e donne, nel confronto cordiale e costruttivo di diverse esperienze e sensibilità;

una Chiesa sinceramente disponibile alla condivisione ecumenica, al dialogo interreligioso, al confronto interculturale; una Chiesa aperta sulla città, cioè inserita nella società, con un'attenzione preferenziale ai poveri.

La Parrocchia - ci ricordano i nostri Vescovi nel documento /I volto missionario delle parrocchie in un mondo che cambia - può incarnare questa nuova immagine esemplare di Chiesa, questa storia nuova di fede, speranza e carità. "La parrocchia - si legge nel documento "Da questo vi riconosceranno"4 sulle caritas parrocchiali - è un segno e un luogo rinnovato di evangelizzazione: uno strumento per dire oggi la Parola di Dio che salva; per dire ancora, con fermezza, pazienza e simpatia col nostro tempo, le ragioni della fede in Gesù Cristo morto e risorto per noi; per accompagnare ogni persona a scoprire la propria filiazione divina" (n. 9).

La Parrocchia è Chiesa tra le case, è comunione di persone: più che una macchina organizzativa è un cuore che vive: "è fatta per tutti e da tutti. Ciascuno è membro, ciascuno è parte, ciascuno è pietra viva di quel tempio - scriverà don Primo Mazzolari - Nessuno può essere escluso. Se c'è una preferenza, questa è per i poveri" ${ }^{5}$. Nella Parrocchia la Chiesa cresce nel suo volto di 'Madre' (Il volto missionario, 7): "nella parrocchia - disse Giovanni Paolo II ai vescovi lombardi nell'incontro-visita ad limina del 18 dicembre 1986 - la Chiesa mostra veramente la maternità rivolta a tutti, senza criteri esclusivi di elitarietà ed impegnandosi ad essere educatrice convinta e fiduciosa di cristiani sempre più aperti allo Spirito".

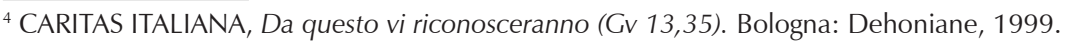

${ }^{5}$ MAZZOLARI..., op. cit. 


\section{I dieci volti della parrocchia 'Chiesa fraterna'}

Incarnazione, comunione, maternità - anche alla luce del documento $\mathrm{CEI}$, Il volto missionario delle parrocchie in un mondo che cambia - sono le parole chiavi sulle quali costruire i volti di una parrocchia oggi come 'Chiesa fraterna'.

Primo volto: pluralità delle culture e delle diverse sensibilità porta a comprendere il volto e lo stile di una 'parrocchia multietnica', cioè 'luogo di incontro tra culture'. Sembrano dover andare in questo senso alcuni 'servizi segno', ma anche alcune esperienze di celebrazione cariche di problemi umani (giustizia, pace, salvaguardia dell' ambiente...). Sul piano interculturale la parrocchia dovrà avere alcune attenzioni nuove e integrate, che derivano anche dai fenomeni della mobilità umana e dell'immigrazione.

Secondo volto: non solo la pluralità delle culture, ma anche la pluralità delle religioni aprono lo stile della parrocchia ad essere 'luogo interreligioso', scegliendo lo stile del dialogo. II Concilio Vaticano II, con il documento Nostra Aetate, Paolo VI nell' enciclica Ecclesiam suam e nell'esortazione Evangelii nuntiandi, Giovanni Paolo II nella lettera apostolica Novo Millennio ineunte e nel messaggio per la pace del 1 gennaio 2001, hanno richiamato il dovere del dialogo interreligioso, come una delle prospettive dell'inculturazione della fede e dell'incontro nuovo tra le religioni. "Nella condizione di più spiccato pluralismo culturale e religioso - ha scritto il Papa polacco nella Novo Millennio ineunte -, quale si va prospettando nella società del nuovo millennio, il dialogo è importante anche per mettere un sicuro presupposto di pace" (n. 55).

Terzo volto: le esperienze positive di dono e di amore, nate dall'ascolto della Parola e alimentate dai sacramenti, che si costruiscono in parrocchia la rendono 'luogo d'amore', 'sacramento' dell'amore tra Dio e l'uomo.

Quarto volto: luogo 'accanto' alle persone, la parrocchia è il primo luogo ecclesiale in cui si impara e si costruisce la 'prossimità' come stile e prospettiva. Una prossimità che chiede nuove forme di accostamento per I'annuncio (la storia di Filippo evocata dagli Atti, ma anche quella di Stefano indicano questa nuova 'diaconia' dell'annuncio), nuovi linguaggi, una nuova valorizzazione degli itinerari di preparazione dei sacramenti, una nuova pastorale d'ambiente.

Quinto volto: luogo 'dentro' il territorio, la parrocchia non è estranea ai problemi della gente, ai luoghi istituzionali in cui cresce la democrazia, anche se non diventa strumento diretto della partecipazione politica. In questa collocazione territoriale matura una scelta profetica della parrocchia, 
che sposa la 'relatività delle cose', cioè la povertà, la 'condivisione delle cose', cioè la colletta, esperienze di servizio sociale esemplari e nuove.

Sesto volto: luogo 'aperto', la parrocchia aiuta a superare i localismi e gli individualismi, a sentire la 'responsabilità di tutti', a educare alla 'mondialità' come uno dei volti della 'cattolicità'. Nella liturgia noi respiriamo questa 'universalità', non sempre la respiriamo nella catechesi e nella carità.

Settimo volto: la parrocchia serve la nota dell'unità della Chiesa cercando non di essere soggetto autoreferenziale, ma di educazione al senso diocesano e universale, tenendo viva la dinamica dell'uno-molti che è propria della Chiesa.

Ottavo volto: la parrocchia ha una 'guida', un 'ministro', non è guidata dal popolo, ma il popolo di Dio chiede al suo interno un 'garante', un 'custode' che richiama continuamente il collegamento all'esperienza apostolica della Chiesa come 'norma normante' la propria vita. Il ruolo del prete va riscoperto 'in parrocchia', dentro una prospettiva ecclesiale di 'ministerialità diffusa', ma anche dentro una prospettiva che valorizza anche la 'sintesi' (parroco ministro della sintesi).

Nono volto: la parrocchia 'preferisce gli ultimi'. Si tratta di riordinare le cose, il tempo, le persone, i luoghi parrocchiali a partire da chi 'manca', da chi è 'lontano', da chi è 'solo', da chi 'soffre', da chi 'cresce', da chi 'non lavora'. Parlare di parrocchia che "preferisce gli ultimi" non significa avere intenzioni 'classiste' o 'populiste': "vuol dire semplicemente - ricordava don Mazzolari - amare di più chi ha bisogno di essere amato di più e non lasciare fuori questi o quelli dal nostro amore ${ }^{\prime \prime}$. Oltre che nelle opere di carità, nei sacramenti - in particolare nel sacramento della misericordia di Dio, ma anche dell'Unzione dei malati - si respira molto quest'aria di attenzione agli ultimi, ai peccatori, a chi ha sbagliato, a chi è malato, a chi è straniero.

Decimo volto: la parrocchia è serva del 'mistero', del 'Santo', dell' 'oltre'. L'Eucarestia che è forma della Chiesa, è forma anche della carità: nel dono, nella memoria delle parole e dei gesti di Gesù che 'passò in mezzo a noi facendo del bene', nel segno di pace, nel servizio. Non perdere la dimensione del 'mistero' è' un aspetto importante per non ridurre la parrocchia semplicemente a un'insieme di servizi e di proposte strutturate rigide, anche sul piano caritatativo. La 'mistica parrocchiale' nasce dall'educazione alla libertà, alla preghiera, al senso della festa e della domenica, al rispetto per ciò che è creduto senza una logica ('religiosità popolare').

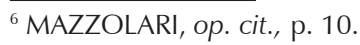




\section{Segni e gesti per un cammino di carità comune in parrocchia}

In questa parrocchia, Chiesa fraterna, che vive la scelta preferenziale dei poveri, nascono alcuni luoghi e spazi importanti per camminare insieme e per coniugare liturgia, catechesi e carità. In questa parrocchia nasce la caritas parrocchiale.

- Per dare voce al Vangelo e per essere tra le persone Chiesa fraterna, in particolare tra chi vive un bisogno con sofferenza, portatori di una Parola, ma anche di un bene, di un dono, la Parrocchia è chiamata a diventare anzitutto un luogo di ascolto, un 'Centro di ascolto'.

La parola è bella, anche perché è condivisa nella Chiesa con una ricchezza di significati. II Centro di ascolto richiama I'ascolto della Parola nella comunità e l'ascolto delle parole dei piccoli - per parafrasare un'espressione di don Primo Mazzolari. Il Centro di ascolto indica la voglia di rischiare la condivisione, offre una disponibilità, costruisce il dialogo, richiama al perdono, costruisce la speranza, prepara la visita alla famiglia, allo straniero.

La "qualità dell'annuncio" al Centro di ascolto passa attraverso la "qualità della testimonianza delle parole", la professionalità delle risposte, l'interesse per l'ascoltatore, la costruzione di una risposta che, anche se non risolutiva, è espressiva di una fraternità. La qualità della testimonianza al Centro di ascolto passa attraverso la capacità non solo di rispondere ai problemi, ma anche di rilanciare in forme educative e provocatorie alla comunità cristiana i problemi e i temi incontrati.

I luoghi di ascolto sono una sfida per la pastorale odierna, chiamata a inventare nuove forme di relazione, di incontro. II centro d'ascolto diventa un luogo importante per rendere 'esperienziale' la catechesi e legare alla quotidianità e al territorio la nostra celebrazione eucaristica domenicale.

- Un secondo luogo sono i segni. I sacramenti, le esperienze, i servizi segno tra i poveri, presto, con intelligenza e amore, sono luoghi importanti sul piano dell'educazione della fede, ma anche della credibilità della fede, che vanno valorizzati insieme. Le esperienze, poi, del volontariato e del servizio civile sono forme importanti di partecipazione e di responsabilità o educative alla partecipazione e alla responsabilità nella Chiesa da valorizzare, perché espressione della speranza laicale.

- Un terzo luogo: la colletta, il dono. Ogni servizio segno vive anche della 'colletta', che è un altro modo di partecipare concretamente alle gioie e alle speranze, alle tristezze e alle angosce del nostro tempo e con la Chiesa, un altro modo per fare comunione. $\mathrm{E}^{\prime}$ un altro segno, la colletta, che si crede nel valore di un segno, di un gesto, di una struttura, di una campagna. 
La colletta, in parrocchia, assume oggi la forma del 'Progetto', della promozione di forme nuove e alternative di mercato e di finanza, del credito gratuito, dell'anticipazione. L'Offertorio, ma anche la proposta catechistica di un tempo forte, si carica di questa 'intelligenza, concretezza della comunione, gestita poi direttamente dalla caritas diocesana.

- Un quarto luogo: I'annuncio e la denuncia. Non si può ingenuamente pensare che ogni male, ogni povertà e abbandono sia frutto soltanto dell'incapacità, della debolezza dell'individuo. Molti mali sono 'strutturali', frutto di un ideologia e di una 'struttura di peccato' che rende I'uomo incapace di gestire la propria vita, le proprie risorse. La denuncia, in questo sempre più complesso mondo della comunicazione e della politica, diventa uno strumento importante di annuncio, mediato dalla dottrina sociale della chiesa, ma anche portato nella preghiera e nella riflessione soprattutto domenicale, per ricordare, ammonire, stimolare nuove progettualità politiche, economiche e sociali sul piano del rispetto della dignità e dei diritti, della politica familiare, della casa, del lavoro, della salute e dei servizi alla persona. Ogni denuncia, per non essere superficiale ed emotiva, deve essere accompagnata dallo studio, dall'osservazione puntuale, dalla discussione fraterna, anche negli organi di partecipazione pastorale. "La denucia, nei cuori profondi, - ricordava don Primo Mazzolari - anche se vivace e ardita, è sempre una pretesa d'amore e un documento di vita"7.

- Un quinto luogo: il tempo e la memoria. La carità interpreta i tempi (è uno dei 'segni dei tempi'), ma si concretizza dentro il 'tempo liturgico'. Quaresima e Avvento sono i tempi che, proprio perchè 'forti', chiedono una attenzione particolare a coniugare fede e vita, contemplazione e azione: per questo i tempi vedono anche una maggiore attenzione a iniziative e percorsi fraterni e di carità. Anche alcune memorie e feste di santi, dentro l'anno liturgico, richiamano la carità come vocazione che incarna concretamente la fede e la speranza.

\section{Conclusioni: I’annuncio ai poveri tra fede e vita}

Il Vangelo della carità, le scelte di vita e il servizio alla Chiesa e al territorio hanno bisogno di un nuovo sforzo per un lavoro pastorale d'insieme, dove l'annuncio ai poveri tra fede e vita si coniugano attorno ad alcuni aspetti fondamentali: itinerari vocazionali, itinerari di formazione alla ministerialità, l'attenzione al territorio, la scelta preferenziale per i poveri.

${ }^{7}$ Ibidem, p. 47. 